\title{
Factors Associated with Insomnia Among Truck Drivers in Japan
}

\author{
Takashi Miyachi' \\ Kyoko Nomura $\mathbb{D}^{2}$ \\ Sachiko Minamizono ${ }^{2}$ \\ Kazuki Sakai' \\ Toyoto Iwata ${ }^{2}$ \\ Yuta Sugano' \\ Shun Sawaguchi ${ }^{3}$ \\ Kouhei Takahashi ${ }^{3}$ \\ Kazuo Mishima $\mathbb{D}^{4}$ \\ 'Department of Medicine, Akita \\ University Graduate School of Medicine, \\ Akita, Japan; ${ }^{2}$ Department of \\ Environmental Health Science and Public \\ Health, Akita University Graduate School \\ of Medicine, Akita, Japan; ${ }^{3}$ Japan Health \\ Insurance Association, Akita Branch, \\ Akita, Japan; ${ }^{4}$ Department of \\ Neuropsychiatry, Akita University \\ Graduate School of Medicine, Akita, \\ Japan
}

Correspondence: Kyoko Nomura Department of Environmental Health Science and Public Health, Akita University Graduate School of Medicine, I-I-I Hondo, Akita, 010-8543, Japan

Tel +8I-I8-884-6086

Fax +8I-I8-836-2609

Email nomurakyoko@gmail.com
Purpose: Truck drivers with insomnia are at a high risk of traffic accidents. We aimed to investigate the prevalence of insomnia and identify its associated factors among truck drivers in Japan.

Methods: Participants were 2927 male truck drivers younger than 65 years old. Selfadministered questionnaires were used to assess insomnia symptoms, State-Trait Anxiety Inventory, drinking, smoking habits, body mass index, caffeine intake, as well as daily driving hours, consecutive days away from home, and driving distance. Insomnia symptoms included difficulty initiating sleep, maintaining sleep and early morning awakening. Insomnia was defined when any of these symptoms were observed with daily tiredness.

Results: The prevalence of insomnia among the subjects was $13.3 \%(n=356)$, of which $13.5 \%$ had difficulty initiating sleep, $78 \%$ had difficulty maintaining sleep, and $26.4 \%$ had early morning awakening. After adjusting for covariates, drinking habits, daily driving hours, and STAI score were significantly and linearly associated with insomnia; the adjusted odds ratio (OR) of drinking habits for insomnia was 1.74 [95\% confidence interval (CI), 1.23-2.47] for heavy drinkers compared to non-drinkers (trend $p<0.001$ ); the adjusted OR of daily driving hours was 1.87 (95\% CI, 1.00-3.49) for 12 hours or longer in a day compared to $<8$ hours in a day (trend $p<0.001)$; the adjusted OR of STAI quartiles was 5.30 (95\% CI, 3.66-7.67) for the highest quartile compared to the lowest quartile (trend $p<0.001$ ).

Conclusion: The present study demonstrated that insomnia is prevalent among truck drivers in Japan, and its risk factors include drinking habits, daily driving hours, and anxiety.

Keywords: insomnia, truck driver, State-Trait Anxiety Inventory, STAI, working environment, lifestyle

\section{Introduction}

The occupation of truck drivers is characterized by high job demands, including long and irregular working hours with low wages. ${ }^{1-3}$ In Japan, a deadly highway bus incident occurred in 2016, wherein 15 people died. This incident later led to a reform in the working style of commercial cargo drivers. ${ }^{4}$ However, according to the Labor Survey 2019, conducted by the Ministry of Health, Labor, and Welfare, the average of annual working hours of truck drivers was 2580 hours, of which as much as $17 \%$ were overtime, compared to other workers who worked 2076 hours with $7.5 \%$ of overtime on average. Despite the overtime work done by truck drivers, their annual income is much lower than the average income of the Japanese working population. ${ }^{5}$

Poor working conditions are largely explained by the unique payment system in which the salary is determined based on the distance driven. ${ }^{6}$ Hence, in order to 
earn a living, drivers are highly motivated to drive long distances. However, such a working style may not last long with the constantly increasing age of drivers, thus ensuring that the workforce is difficult. Consequently, the number of drivers declined from 979,996 to 767,310 between 1995 and $2015^{7}$ despite the rapid increase in transportation demands, which has expanded during and post the COVID-19 periods. ${ }^{8}$ The shortage of truck drivers further worsens the working environment of the currently working truck drivers.

The poor working environment of drivers may be associated with disturbed sleep quality and quantity, causing daytime sleepiness and incidents of falling asleep while driving, resulting in fatal tragic traffic accidents. ${ }^{9-11}$ According to the annual report of the Transport Department of the National Police Agency, 3133 people were killed in road accidents in 2019, of which $265(8.5 \%)$ cases were involved with trucks. ${ }^{12}$ More than $80 \%$ of these accidents were triggered by driver inattention, misidentification, or operational errors that might be accounted for by symptoms of insomnia. ${ }^{13}$ Insomnia is a common, patient-reported complaint that is characterized by difficulty falling asleep, maintaining sleep, or premature awakening. ${ }^{14}$ Untreated insomnia causes many difficulties for patients in terms of increased fatigue, reduced quality of life (QOL), impaired daytime functioning, and an increased risk of accidents and injuries. ${ }^{15-17}$

Lifestyle of truck drivers such as smoking, drinking, enforced sedentarism, and unhealthy eating habits may also affect the quality and quantity of sleep, which is further associated with cardiovascular disease. ${ }^{18,19}$ Truck drivers prefer to consume caffeine as a countermeasure to sleepiness at the wheel, which may in turn worsen sleep quality. ${ }^{20}$ In addition, truck drivers are subjected to psychological distress and anxiety due to chronic time pressures, continuous absence from home, and disrespectful treatment from others. ${ }^{21-23}$ Indeed, weather and road conditions are identified as risk factors for traffic accidents, which may enhance driver anxiety. ${ }^{24}$ A previous study revealed that $25.9 \%$ of truck drivers reported feeling anxious. ${ }^{25}$ Such a mental health problem of truck drivers are closely related to sleep problems. ${ }^{26}$ Despite these previous findings, there are very few comprehensive epidemiological studies investigating insomnia taking into account the conventionally suggested factors. Thus, the purpose of the present study was to investigate factors associated with insomnia among truck drivers in Japan.

\section{Methods}

\section{Participants}

We asked the Japan Truck Association, Akita prefectural branch to recruit drivers to participate in this study via the Japan Health Insurance Association, Akita branch. At the time of investigation in July 2020, companies belonging to the Japan Truck Association, Akita prefectural branch had 7200 trucks in total. Thus, we distributed the same numbers of the written informed consent embedded in the selfadministered questionnaire. We received responses from 2927 drivers who agreed to participate in the study. Although the Truck Association does not register the number of drivers, according to the latest Population Census in 2015, ${ }^{27}$ there were 5950 truck drivers in Akita Prefecture. The response rate was estimated to be $49.2 \%$. Participants were excluded if they were females ( $\mathrm{n}=60), 65$ years or older $(\mathrm{n}=168)$, non-truck drivers including office workers $(\mathrm{n}=11)$, and those who did not respond to a question related to insomnia symptoms $(n=8)$. Consequently, 2685 participants were included in the analyses.

This study was approved by the Medical Ethical Committee of the University of Akita (Number 2456) and conducted in accordance with the Declaration of Helsinki.

\section{Outcome}

We defined insomnia based on the International Classification of Sleep Disorders, third edition (ICSD3 ), diagnostic criteria for chronic insomnia disorder. ${ }^{28}$ Study subjects were asked if they experienced any of the following three insomnia symptoms (ie, difficulty initiating sleep, difficulty maintaining sleep, and episode of early morning awakening) that occurred at least three times per week over a period of three months. Difficulty initiating sleep was described as difficulty falling asleep at the beginning of the night and even after spending two hours in bed. Difficulty maintaining sleep was described as an inability to stay asleep through the night, waking up at least twice during the night. Early morning awakening involved waking up two hours before a person wanted or planned to wake up in the morning and struggling to get back to sleep. Previously we knew that those who had initiation difficulty are more likely to have mental health conditions such as depression and anxiety, ${ }^{29,30}$ we defined sleep initiation difficulty even if the subjects had both initiation and other insomnia 
symptoms. In addition, since difficulty maintaining sleep and early morning awakening were difficult to discriminate from each other, and considering that there are very small numbers who manifested these two conditions, we treated them simultaneously and defined them as sleep maintenance difficulty in further analyses. Insomnia and its subtypes (sleep initiation difficulty and sleep maintenance difficulty) were further defined as experiencing daytime tiredness. Daytime tiredness was measured by asking, "Do you still feel tired and fatigued the next day?" Responses were based on the frequency of a 4-point Likert scale (never, rarely, often, always) and accordingly subjects who answered "often/always" were considered to have daytime tiredness.

\section{STAI}

Anxiety was measured using a validated Japanese version of the State-Trait Anxiety Inventory (STAI), which is the most commonly used clinical anxiety rating scale. ${ }^{31,32}$ This was developed in two forms: state anxiety and trait anxiety. In this study, in order to identify anxiety pertaining to the working environment among truck drivers, the state of anxiety was used, which is defined as a transitory emotional response to a stressful situation that involves unpleasant feelings of tension and apprehensive thoughts. We asked the subjects to respond to the STAI before they started driving trucks. Response pattern was measured based on a 4-point Likert scale ranging from "almost never" (1) to "almost always" (4), and sum of the score was divided into a quartile category.

\section{Caffeine Intake}

We investigated caffeinated beverages and foods consumption: tea, including green tea, Oolong tea (semi-fermented tea), black tea (fermented tea), coffee, energy drinks, foods including menthol gum, caffeine drop, and other caffeinated tablets. The drinks were measured by the number of cups consumed (one cup was converted into $150 \mathrm{~mL}$ ). The caffeine consumption of each food and beverage was calculated according to the Food Safety Commission of Japan. ${ }^{33}$ We asked the participants about their weekly intake frequency and the daily amount of each caffeinated food and beverage consumed. Weekly caffeine consumption was then calculated by multiplying the daily caffeine intake and frequency on a weekly basis. The subjects were then divided into two groups (normal: $\leq 2800 \mathrm{mg} /$ week vs heavy: $>2800 \mathrm{mg} /$ week). ${ }^{34}$

\section{Driving Distance}

Drivers were categorized into three groups, long-haul, middle-haul, and short-haul, based on the area in which the drivers worked. Japan consists of 47 prefectures divided into five regions, and Akita falls within one region. For example, long-haul indicated a region without boundaries (ie, a whole country), middle-haul indicated the Tohoku region (one of the five regions), and shorthaul indicated the Akita prefecture.

\section{Covariates}

Other covariates investigated were age, average daily sleep duration, drinking habits (non-drinker vs normal: ethanol consumption $253 \mathrm{~g}$ or lesser/week vs heavy: ethanol consumption $253 \mathrm{~g}$ or larger/week), smoking habits (current vs past vs never), Body Mass Index (BMI; underweight: smaller than $18.5 \mathrm{~kg} / \mathrm{m}^{2}$ vs normal: $18.5-24.9 \mathrm{~kg} / \mathrm{m}^{2}$ vs obese: 25 or larger $\left.\mathrm{kg} / \mathrm{m}^{2}\right)$, daily driving hours ( $<8$ hours vs $8-9$ hours vs $10-11$ hours vs $\geq 12$ hours), and consecutive days away from home (not applicable vs one day vs two days or longer).

\section{Statistical Analyses}

A $t$-test or chi-square test was used to investigate the association between insomnia and covariables according to the distribution of each variable. Factors associated with insomnia, sleep initiation difficulty, and sleep maintenance difficulty were identified using a logistic regression model. Odds ratios (ORs) were computed along with 95 confidence intervals (95\% CIs). Multivariable logistic analysis was used to adjust for significant variables $(p<0.25)$ in univariate logistic models. Trend $p$ for linearity was investigated for drinking habits, working status of daily driving hours, and STAI score.

All statistical analyses were performed using $\mathrm{R}$ version software (3.6.1). All tests were two-sided, and statistical significance was set at $p<0.05$.

\section{Results}

\section{Baseline Characteristics of Male Truck Drivers According to Insomnia and Its Subtypes (See Table I)}

Among 2685 truck drivers who completed the questionnaires, the mean age was $47.9 \pm 9.5$ years, and the mean STAI score was $44.7 \pm 8.3$ points. The prevalence of insomnia was $13.3 \%$, of which 48 subjects had sleep initiation difficulty and 308 subjects had sleep maintenance difficulty. 
Table I Baseline Characteristics of Male Truck Drivers According to Insomnia and Its Subtypes

\begin{tabular}{|c|c|c|c|c|c|c|c|c|c|c|c|c|c|}
\hline & \multirow{3}{*}{\multicolumn{2}{|c|}{$\begin{array}{l}\text { Total } \\
(N=2685)\end{array}$}} & \multicolumn{4}{|c|}{ Insomnia } & \multirow{3}{*}{$p$ value** } & \multicolumn{6}{|c|}{ Insomnia Subtypes } \\
\hline & & & \multicolumn{2}{|l|}{$(+)$} & \multicolumn{2}{|l|}{$(-)$} & & \multicolumn{3}{|c|}{$\begin{array}{l}\text { Sleep Initiation } \\
\text { Difficulty }\end{array}$} & \multicolumn{3}{|c|}{$\begin{array}{l}\text { Sleep Maintenance } \\
\text { Difficulty }\end{array}$} \\
\hline & & & \multicolumn{2}{|c|}{$\begin{array}{l}(N=356 \\
\text { I3.26\%) }\end{array}$} & \multicolumn{2}{|c|}{$\begin{array}{l}(N=2329 ; \\
86.74 \%)\end{array}$} & & \multicolumn{2}{|c|}{$N=48$} & $p$ value** & \multicolumn{2}{|c|}{$N=308$} & $p$ value $* *$ \\
\hline Age years* & \multicolumn{2}{|c|}{$47.9 \pm 9.5$} & \multicolumn{2}{|c|}{$48.5 \pm 9.0$} & \multicolumn{2}{|c|}{$47.9 \pm 9.6$} & 0.229 & \multicolumn{2}{|c|}{$47.4 \pm 7.6$} & 0.920 & \multicolumn{2}{|c|}{$48.7 \pm 9.2$} & 0.154 \\
\hline $\begin{array}{l}\text { Average daily sleep } \\
\text { hours* }\end{array}$ & \multicolumn{2}{|c|}{$6.73 \pm 1.13$} & \multicolumn{2}{|c|}{$6.35 \pm 1.20$} & \multicolumn{2}{|c|}{$6.79 \pm 1.11$} & $<0.0001$ & \multicolumn{2}{|c|}{$5.67 \pm 1.34$} & $<0.0001$ & \multicolumn{2}{|c|}{$6.46 \pm 1.15$} & $<0.0001$ \\
\hline $\begin{array}{l}\text { Drinking habits (Ethanol } \\
\text { consumption g/week*) }\end{array}$ & \multicolumn{2}{|c|}{$2|4 \pm 20|$} & \multicolumn{2}{|c|}{$246 \pm 210$} & \multicolumn{2}{|c|}{$209 \pm 199$} & & \multicolumn{2}{|c|}{$170 \pm 197$} & & \multicolumn{2}{|c|}{$258 \pm 209$} & \\
\hline Non-drinker & 659 & $(25.1)$ & 66 & $(18.6)$ & 593 & $(26.2)$ & 0.006 & 14 & $(29.2)$ & 0.466 & 52 & $(17.0)$ & 0.001 \\
\hline Normal ( $\leq 253 \mathrm{~g} /$ week) & 1004 & $(38.3)$ & 139 & $(39.3)$ & 865 & $(38.2)$ & & 21 & $(43.8)$ & & 118 & $(38.6)$ & \\
\hline Heavy (>253 g/week) & 958 & (36.6) & 149 & $(42.1)$ & 809 & $(35.7)$ & & 13 & (27.1) & & 136 & $(44.4)$ & \\
\hline $\begin{array}{l}\text { Caffeine intake (mg/ } \\
\text { week*) }\end{array}$ & \multicolumn{2}{|c|}{$1470 \pm 1220$} & \multicolumn{2}{|c|}{$1540 \pm 1180$} & $1460 \pm$ & 220 & & 158 & \pm 1087 & & 1534 & 1197 & \\
\hline $\begin{array}{l}\text { Normal ( } \leq 2800 \mathrm{mg} / \\
\text { week) }\end{array}$ & 2427 & $(90.4)$ & 317 & $(89.0)$ & 2110 & $(90.6)$ & 0.355 & 43 & (89.6) & 0.812 & 274 & $(89.0)$ & 0.36 \\
\hline $\begin{array}{l}\text { Heavy (>2800 mg/ } \\
\text { week) }\end{array}$ & 258 & (9.6) & 39 & $(I I .0)$ & 219 & $(9.4)$ & & 5 & $(10.4)$ & & 34 & $(11.0)$ & \\
\hline Never smoker & 321 & $(12.1)$ & 32 & $(9.1)$ & 289 & $(12.6)$ & 0.099 & 2 & $(4.2)$ & 0.032 & 30 & $(9.8)$ & 0.069 \\
\hline Past smoker & 826 & $(3 \mid .2)$ & 122 & $(34.6)$ & 704 & $(30.7)$ & & 10 & (20.8) & & 112 & $(36.7)$ & \\
\hline Current smoker & 1502 & $(56.7)$ & 199 & $(56.4)$ & 1303 & $(56.8)$ & & 36 & $(75.0)$ & & 163 & $(53.4)$ & \\
\hline $\begin{array}{l}\text { Body-mass index }(\mathrm{kg} / \\
\left.\mathrm{m}^{2 *}\right)\end{array}$ & $24.5 \pm$ & & 24.7 & & $24.5 \pm$ & & & 24.4 & 44.0 & & 24.7 & & \\
\hline $\begin{array}{l}\text { Underweight }(<18.5 \mathrm{~kg} / \\
\left.\mathrm{m}^{2}\right)\end{array}$ & 60 & (2.3) & 12 & (3.4) & 48 & $(2.1)$ & 0.313 & 3 & $(6.3)$ & 0.146 & 9 & $(3.0)$ & 0.64 \\
\hline $\begin{array}{l}\text { Normal }(18.5-24.9 \mathrm{~kg} / \\
\left.\mathrm{m}^{2}\right)\end{array}$ & 1542 & $(58.7)$ & 203 & $(57.5)$ & 1339 & $(58.8)$ & & 26 & $(54.2)$ & & 177 & $(58.0)$ & \\
\hline Obese $\left(\geq 25 \mathrm{~kg} / \mathrm{m}^{2}\right)$ & 1027 & $(39.1)$ & 138 & $(39.1)$ & 889 & $(39.1)$ & & 19 & (39.6) & & 119 & $(39.0)$ & \\
\hline Daily driving $<8$ hours & 1455 & $(55.3)$ & 173 & $(48.9)$ & 1282 & (56.4) & 0.001 & 23 & (48.9) & 0.023 & 150 & (48.9) & 0.004 \\
\hline 8-9 hours & 806 & $(30.7)$ & 109 & $(30.8)$ & 697 & $(30.6)$ & & 11 & (23.4) & & 98 & $(31.9)$ & \\
\hline 10-11 hours & 278 & $(10.6)$ & 51 & $(14.4)$ & 227 & $(10.0)$ & & 11 & (23.4) & & 40 & $(13.0)$ & \\
\hline$\geq 12$ hours & 90 & (3.4) & 21 & (5.9) & 69 & (3.0) & & 2 & $(4.3)$ & & 19 & $(6.2)$ & \\
\hline $\begin{array}{l}\text { Driving distance: Short- } \\
\text { haul (Akita prefecture) }\end{array}$ & 1600 & $(60.5)$ & 189 & $(53.1)$ & 1411 & $(6 \mid .6)$ & 0.008 & 22 & (45.8) & 0.043 & 167 & (54.2) & 0.043 \\
\hline $\begin{array}{l}\text { Middle-haul (Tohoku } \\
\text { region) }\end{array}$ & 425 & $(16.1)$ & 71 & $(19.9)$ & 354 & (15.5) & & 13 & (27.1) & & 58 & $(18.8)$ & \\
\hline $\begin{array}{l}\text { Long-haul (no } \\
\text { boundary) }\end{array}$ & 620 & (23.4) & 96 & $(27.0)$ & 524 & (22.9) & & 13 & (27.1) & & 83 & $(27.0)$ & \\
\hline
\end{tabular}

(Continued) 
Table I (Continued).

\begin{tabular}{|c|c|c|c|c|c|c|c|c|c|c|c|c|c|}
\hline & \multirow{3}{*}{\multicolumn{2}{|c|}{$\begin{array}{l}\text { Total } \\
(N=2685)\end{array}$}} & \multicolumn{4}{|c|}{ Insomnia } & \multirow{4}{*}{\begin{tabular}{|l|}
$p$ value** \\
0.003 \\
\end{tabular}} & \multicolumn{6}{|c|}{ Insomnia Subtypes } \\
\hline & & & \multicolumn{2}{|l|}{$(+)$} & \multicolumn{2}{|l|}{$(-)$} & & \multicolumn{3}{|c|}{$\begin{array}{l}\text { Sleep Initiation } \\
\text { Difficulty }\end{array}$} & \multicolumn{3}{|c|}{$\begin{array}{l}\text { Sleep Maintenance } \\
\text { Difficulty }\end{array}$} \\
\hline & & & \multicolumn{2}{|c|}{$\begin{array}{l}(N=356 \\
\text { I3.26\%) }\end{array}$} & \multicolumn{2}{|c|}{$\begin{array}{l}(N=2329 \\
86.74 \%)\end{array}$} & & \multicolumn{2}{|c|}{$N=48$} & \multirow{2}{*}{$\begin{array}{l}\text { P value** } \\
0.203\end{array}$} & \multicolumn{2}{|c|}{$N=308$} & \multirow{2}{*}{$\begin{array}{l}\text { p value** } \\
0.01\end{array}$} \\
\hline $\begin{array}{l}\text { Consecutive days away } \\
\text { from home: Not } \\
\text { applicable }\end{array}$ & 1769 & $(69.0)$ & 221 & $(64.2)$ & 1548 & $(69.8)$ & & 29 & $(63.0)$ & & 192 & $(64.4)$ & \\
\hline One day & 301 & $(11.7)$ & 34 & (9.9) & 267 & $(12.0)$ & & 4 & (8.7) & & 30 & $(10.1)$ & \\
\hline Two days or longer & 493 & $(19.2)$ & 89 & $(25.9)$ & 404 & $(18.2)$ & & 13 & $(28.3)$ & & 76 & $(25.5)$ & \\
\hline $\begin{array}{l}\text { State-Trait Anxiety } \\
\text { Inventory (STAI) Score* }\end{array}$ & \multicolumn{2}{|c|}{$44.7 \pm 8.3$} & \multicolumn{2}{|c|}{$49.9 \pm 9.1$} & \multicolumn{2}{|c|}{$43.9 \pm 7.9$} & & \multicolumn{2}{|c|}{$54.4 \pm 8.8$} & & \multicolumn{2}{|c|}{$49.1 \pm 8.9$} & \\
\hline \multicolumn{14}{|l|}{ Quartile } \\
\hline$\leq 39$ & 642 & (25.7) & 44 & $(13.2)$ & 598 & $(27.6)$ & $<0.0001$ & 2 & $(4.4)$ & $<0.0001$ & 42 & $(14.6)$ & $<0.0001$ \\
\hline 40 to $\leq 44$ & 629 & $(25.2)$ & 53 & (I5.9) & 576 & $(26.6)$ & & 3 & (6.5) & & 50 & $(17.4)$ & \\
\hline 45 to $\leq 50$ & 679 & $(27.2)$ & 86 & $(25.8)$ & 593 & $(27.4)$ & & 13 & $(28.3)$ & & 73 & $(25.4)$ & \\
\hline$\geq 51$ & 548 & $(21.9)$ & $|5|$ & $(45.2)$ & 397 & $(18.4)$ & & 28 & $(60.9)$ & & 123 & $(42.7)$ & \\
\hline
\end{tabular}

Notes: *Plus-minus values are means \pm standard deviation. Numbers and percentages are shown for categorical variables. $* *$ t-test for continuous variables or chi-square test for categorial variables.

Truck drivers with insomnia were more likely to be heavy alcohol drinkers, long-haul drivers, have longer driving hours, and have many consecutive days away from home than those without insomnia. In addition, the prevalence of insomnia was significantly higher among drivers with higher STAI scores. Compared with drivers who did not have insomnia, truck drivers with sleep initiation difficulty were more likely to be current smokers, long-haul drivers, have longer driving hours, and have higher STAI scores, whereas truck drivers with sleep maintenance difficulty were more likely to be heavy drinkers, long-haul drivers, have long driving hours, have many consecutive days away from home, and higher STAI scores (Table 1).

\section{Logistic Regression Analyses of Insomnia and Its Subtypes}

Univariate logistic regression analyses demonstrated that significant factors associated with insomnia and sleep maintenance difficulty were drinking habits, smoking habits, daily driving hours, driving distance, consecutive days away from home, and STAI score; significant factors associated with sleep initiation difficulty were smoking habits, daily driving hours, driving distance, consecutive days away from home, and STAI score.

The results of the multiple logistic regression analyses of insomnia, sleep initiation difficulty, and sleep maintenance difficulty are shown in Table 2. After adjusting for covariates, factors statistically associated with insomnia were drinking habits, daily driving hours, and anxiety with linear associations although the second lowest category of driving hour and STAI score were not significant with a reference of the lowest category; adjusted OR of drinking habits was 1.51 (95\% CI, 1.07-2.14) for normal drinker and 1.74 (95\% CI, 1.23-2.47) for heavy drinker compared to non-drinker (trend $p<0.001$ ); adjusted OR of daily driving hours was 1.61 (95\% CI, 1.06-2.43) for 10-11 hours a day and 1.87 (95\% CI, 1.00-3.49) for 12 hours or longer a day compared to $<8$ hours a day (trend $p=0.007$ ); adjusted OR of STAI quartiles was 1.88 (95\% CI, 1.27-2.77) for the second highest quartile and 5.30 (95\% CI, 3.66-7.67) for the highest quartile compared to the lowest quartile (trend $p<0.001$ ). In addition, smoking habits was also statistically associated with insomnia; adjusted OR of past smokers was 1.67 (95\% CI, 1.05-2.66) compared to that of never smokers. On the other hand, there were no significant associations observed with caffeine intake, BMI and other 
Table 2 Logistic Regression Analyses of Insomnia and Its Subtypes

\begin{tabular}{|c|c|c|c|c|c|c|c|c|c|}
\hline & \multicolumn{3}{|c|}{ Insomnia } & \multicolumn{3}{|c|}{ Sleep Initiation Difficulty } & \multicolumn{3}{|c|}{ Sleep Maintenance Difficulty } \\
\hline & $\begin{array}{l}\text { Crude } \\
\text { OR }\end{array}$ & $p$ value** & $\begin{array}{c}\text { Adjusted OR } \\
(95 \% \mathrm{Cl})^{\mathrm{a}}\end{array}$ & $\begin{array}{c}\text { Crude } \\
\text { OR }\end{array}$ & $p$ value** & $\begin{array}{c}\text { Adjusted OR } \\
(95 \% \mathrm{Cl})^{\mathrm{b}}\end{array}$ & $\begin{array}{c}\text { Crude } \\
\text { OR }\end{array}$ & $p$ value $* *$ & $\begin{array}{c}\text { Adjusted OR } \\
(95 \% \mathrm{Cl})^{\mathrm{a}}\end{array}$ \\
\hline Age & 1.01 & 0.229 & $\begin{array}{c}1.00 \\
(0.99-1.02)\end{array}$ & 0.995 & 0.738 & $\begin{array}{c}0.999 \\
(0.97-1.03)\end{array}$ & 1.009 & 0.154 & $\begin{array}{c}1.01 \\
(0.99-1.02)\end{array}$ \\
\hline \multicolumn{2}{|l|}{ Drinking habits* } & 0.006 & & & 0.471 & & & 0.001 & \\
\hline non-drinker & ref & & ref & ref & & & ref & & ref \\
\hline $\begin{array}{l}\text { normal }(\leq 253 \\
\text { g/week) }\end{array}$ & 1.44 & & $\begin{array}{c}1.51 \\
(1.07-2.14)\end{array}$ & 1.03 & & & 1.56 & & $\begin{array}{c}1.63 \\
(1.12-2.38)\end{array}$ \\
\hline $\begin{array}{l}\text { heavy (>253 g/ } \\
\text { week) }\end{array}$ & 1.66 & & $\begin{array}{c}1.74 \\
(1.23-2.47)\end{array}$ & 0.68 & & & 1.92 & & $\begin{array}{c}2.05 \\
(I .4 I-3.00)\end{array}$ \\
\hline \multicolumn{2}{|l|}{ Caffeine intake } & 0.355 & & & 0.812 & & & 0.360 & \\
\hline $\begin{array}{l}\text { normal } \\
(\leq 2800 \mathrm{mg} / \\
\text { week })\end{array}$ & ref & & & & ref & & & ref & \\
\hline $\begin{array}{l}\text { heavy (> } \\
2800 \mathrm{mg} / \text { week) }\end{array}$ & 1.19 & & & 1.12 & & & 1.20 & & \\
\hline \multicolumn{2}{|l|}{ Smoking habits } & 0.101 & & & 0.042 & & & 0.071 & \\
\hline Never & ref & & ref & ref & & ref & ref & & ref \\
\hline Past & 1.56 & & $\begin{array}{c}1.67 \\
(1.05-2.66)\end{array}$ & 2.05 & & $\begin{array}{c}2.26 \\
(0.47-10.83)\end{array}$ & 1.53 & & $\begin{array}{c}1.28 \\
(0.8 \mathrm{I}-2.02)\end{array}$ \\
\hline Current & 1.38 & & $\begin{array}{c}1.50 \\
(0.96-2.34)\end{array}$ & 3.99 & & $\begin{array}{c}4.34 \\
(1.01-18.56)\end{array}$ & 1.21 & & $\begin{array}{c}1.59 \\
(0.98-2.56)\end{array}$ \\
\hline \multicolumn{2}{|l|}{ Body-mass index } & 0.990 & & & 0.941 & & & 0.988 & \\
\hline $\begin{array}{l}\text { non obese } \\
\left(<24.9 \mathrm{~kg} / \mathrm{m}^{2}\right)\end{array}$ & ref & & & ref & & & ref & & \\
\hline $\begin{array}{l}\text { obese }(\geq 25 \mathrm{~kg} / \\
\left.\mathrm{m}^{2}\right)\end{array}$ & 1.00 & & & 1.02 & & & 1.00 & & \\
\hline \multicolumn{2}{|c|}{ Daily driving hours* } & 0.001 & & & 0.032 & & & 0.005 & \\
\hline$<8$ hours & ref & & ref & ref & & ref & ref & & ref \\
\hline $8-9$ hours & 1.16 & & $\begin{array}{c}\text { I.I4 } \\
(0.84-1.54)\end{array}$ & 0.88 & & $\begin{array}{c}0.72 \\
(0.32-1.6 I)\end{array}$ & 1.20 & & $\begin{array}{c}1.21 \\
(0.88-1.66)\end{array}$ \\
\hline $10-11$ hours & 1.67 & & $\begin{array}{c}1.6 \mathrm{I} \\
(1.06-2.43)\end{array}$ & 2.70 & & $\begin{array}{c}2.65 \\
(1.10-6.39)\end{array}$ & 1.51 & & $\begin{array}{c}1.45 \\
(0.93-2.27)\end{array}$ \\
\hline$\geq 12$ hours & 2.26 & & $\begin{array}{c}1.87 \\
(1.00-3.49)\end{array}$ & 1.62 & & $\begin{array}{c}1.12 \\
(0.22-5.74)\end{array}$ & 2.35 & & $\begin{array}{c}1.97 \\
(1.03-3.76)\end{array}$ \\
\hline \multicolumn{2}{|l|}{ Driving distance } & 0.008 & & & 0.049 & & & 0.043 & \\
\hline $\begin{array}{l}\text { short-haul } \\
\text { (Akita } \\
\text { prefecture) }\end{array}$ & ref & & ref & ref & & ref & ref & & ref \\
\hline
\end{tabular}

(Continued) 
Table 2 (Continued).

\begin{tabular}{|c|c|c|c|c|c|c|c|c|c|}
\hline & \multicolumn{3}{|c|}{ Insomnia } & \multicolumn{3}{|c|}{ Sleep Initiation Difficulty } & \multicolumn{3}{|c|}{ Sleep Maintenance Difficulty } \\
\hline & $\begin{array}{l}\text { Crude } \\
\text { OR }\end{array}$ & $p$ value** & $\begin{array}{c}\text { Adjusted OR } \\
(95 \% \mathrm{CI})^{\mathrm{a}}\end{array}$ & $\begin{array}{l}\text { Crude } \\
\text { OR }\end{array}$ & $p$ value** & $\begin{array}{l}\text { Adjusted OR } \\
(95 \% \mathrm{Cl})^{\mathrm{b}}\end{array}$ & $\begin{array}{l}\text { Crude } \\
\text { OR }\end{array}$ & $p$ value** & $\begin{array}{l}\text { Adjusted OR } \\
\qquad(95 \% \mathrm{CI})^{\mathrm{a}}\end{array}$ \\
\hline $\begin{array}{l}\text { middle-haul } \\
\text { (Tohoku region) }\end{array}$ & 1.50 & & $\begin{array}{c}1.22 \\
(0.83-1.79)\end{array}$ & 2.36 & & $\begin{array}{c}2.61 \\
(1.13-6.02)\end{array}$ & 1.38 & & $\begin{array}{c}1.08 \\
(0.7 I-1.65)\end{array}$ \\
\hline $\begin{array}{l}\text { long-haul (no } \\
\text { boundary) }\end{array}$ & 1.37 & & $\begin{array}{c}0.86 \\
(0.49-1.50)\end{array}$ & 1.59 & & $\begin{array}{c}1.34 \\
(0.35-5.08)\end{array}$ & 1.34 & & $\begin{array}{c}0.83 \\
(0.46-1.51)\end{array}$ \\
\hline \multicolumn{2}{|c|}{$\begin{array}{l}\text { Consecutive days away } \\
\text { from home }\end{array}$} & 0.003 & & & 0.212 & & & 0.010 & \\
\hline not applicable & ref & & ref & ref & & ref & ref & & ref \\
\hline one day & 0.89 & & $\begin{array}{c}0.91 \\
(0.54-1.52)\end{array}$ & 0.80 & & $\begin{array}{c}0.55 \\
(0.15-2.11)\end{array}$ & 0.91 & & $\begin{array}{c}0.98 \\
(0.57-1.7 I)\end{array}$ \\
\hline $\begin{array}{l}\text { two days or } \\
\text { longer }\end{array}$ & 1.54 & & $\begin{array}{c}1.52 \\
(0.89-2.61)\end{array}$ & 1.72 & & $\begin{array}{c}1.13 \\
(0.33-3.89)\end{array}$ & 1.52 & & $\begin{array}{c}1.59 \\
(0.89-2.82)\end{array}$ \\
\hline \multicolumn{10}{|l|}{ STAI score* } \\
\hline Quartiles & & $<0.000$ I & & & $<0.0001$ & & & $<0.000$ I & \\
\hline$\leq 39$ & ref & & ref & ref & & ref & ref & & ref \\
\hline 40 to $\leq 44$ & 1.25 & & $\begin{array}{c}1.20 \\
(0.79-1.84)\end{array}$ & 1.56 & & $\begin{array}{c}1.55 \\
(0.26-9.35)\end{array}$ & 1.24 & & $\begin{array}{c}1.18 \\
(0.76-1.82)\end{array}$ \\
\hline 45 to $\leq 50$ & 1.97 & & $\begin{array}{c}1.88 \\
(1.27-2.77)\end{array}$ & 6.56 & & $\begin{array}{c}6.05 \\
(1.34-27.27)\end{array}$ & 1.75 & & $\begin{array}{c}1.67 \\
(I .11-2.5 I)\end{array}$ \\
\hline$\geq 51$ & 5.17 & & $\begin{array}{c}5.30 \\
(3.66-7.67)\end{array}$ & 21.1 & & $\begin{array}{c}22.12 \\
(5.19-94.27)\end{array}$ & 4.41 & & $\begin{array}{c}4.49 \\
(3.06-6.59)\end{array}$ \\
\hline
\end{tabular}

Notes: *Trend $p<0.01$. **Based on wald chi-square statistics. ${ }^{a}$ Adjusted covariates include age, smoking habits, drinking habits, daily driving hours, driving distance, consecutive days away from home, and STAl score. 'Adjusted covariates include age, smoking habits, daily driving hours, driving distance, consecutive days away from home, and STAI score.

working conditions such as driving distance and consecutive days away from home.

When analyses were stratified by two insomnia subtypes, factors associated with sleep initiation difficulty included smoking habits, daily driving hours, driving distance, and anxiety although a linear association was only observed in STAI except for the second lowest quartile. Adjusted OR of current smokers was 4.34 (95\% CI, 1.01-18.56) compared to that of never smokers; adjusted OR of daily driving hours was 2.65 (95\% CI, $1.10-6.39$ ) for $10-11$ hours a day compared to $<8$ hours a day; adjusted OR of driving distance was $2.61(95 \%$ CI, 1.13-6.02) for middle-haul drivers compared to shorthaul drivers; the adjusted OR of STAI quartile was 6.05 (95\% CI,1.34-27.27) for the second highest quartile and
22.12 (95\% CI, 5.19-94.27) for the highest quartile compared to the lowest quartile.

Factors associated with sleep maintenance difficulty were drinking habits, daily driving hours, and anxiety with linear associations, except for the second and third lowest categories of driving hours and second lowest category of STAI score; adjusted OR of drinking habits was $1.63(95 \%$ CI, 1.12-2.38) for normal drinkers and 2.05 (95\% CI, 1.41-3.00) for heavy drinkers compared to non-drinkers (trend $p<0.001$ ); adjusted OR of daily driving hours was 1.97 (95\% CI, 1.03-3.76) for 12 hours or longer a day compared to $<8$ hours a day; adjusted OR of STAI quartiles was 1.67 (95\% CI, 1.11-2.51) for the second highest quartile and 4.49 (95\% CI, 3.06-6.59) for the highest quartile compared to the lowest quartile (trend $p<0.001$ ). 


\section{Discussion}

The present study investigated the prevalence of insomnia and identified its associated factors among Japanese truck drivers. We demonstrated that alcohol consumption, smoking habits, long working hours, and anxiety were mutually associated with insomnia as well as sleep maintenance difficulty except smoking habits, whereas sleep initiation difficulty was associated with smoking habits, long working hours, driving distance, and anxiety. Here, we discuss our interpretation in reference to the previous literature.

In the present study, the prevalence of insomnia among truck drivers was $13.6 \%$, which is much higher than the previously reported $3.2 \%$ among the Japanese general population. ${ }^{35}$ A high prevalence of insomnia in truck drivers has also been observed in Korea, with a prevalence of $15.2 \%$ in Korean truck drivers, compared to $4.1 \%$ among the Korean general population. ${ }^{36}$ Although these studies used different criteria from ours, the high prevalence of insomnia in commercial drivers has received little attention. On the other hand, obstructive sleep apnea (OSA) has been widely recognized following an accident caused by a bullet train driver in 2003, who was later diagnosed with OSA. Consequently, the Ministry of Land, Infrastructure, and Transport distributed a Sleep Apnea Syndrome manual to relevant bodies all over Japan to identify OSA and terminate cargo accidents. ${ }^{37}$ Since then, researchers have conducted epidemiological studies focusing on OSA associated with daytime sleepiness among Japanese truck drivers as a high-risk group for traffic accidents. ${ }^{38,39}$ In this regard, the present study may help relevant people as well as policy makers to know that insomnia is also prevalent among truck drivers, requiring the necessity of epidemiological studies conducted for intervention strategies.

Our result showed that a higher level of anxiety was significantly associated with insomnia of truck drivers even though it requires careful interpretation since the cross-sectional nature of the study does not allow to clarify the causal relationship. ${ }^{40}$ We used the STAI to measure anxiety, which is used in most studies that investigate anxious characteristics in non-clinical samples. ${ }^{41}$ State anxiety refers to transitory unpleasant feelings of apprehension, tension, nervousness, or worry, often accompanied by activation of the autonomic nervous system; it reflects how threatening a person perceives his environment to be. In this regard, STAI scores among truck drivers may reflect emotions at the wheel, worry about time constraints, weather and road conditions, long-time self-confinement during driving, and so on. Although we were not able to investigate personal reasons for anxiety, such as life events or human relationships, our subjects were in full-time labor, indicating that they were a healthy population. Accordingly, the mean STAI score among our truck drivers was $44.7 \pm 8.3$, which was close to the score among previously reported Japanese male workers. ${ }^{42}$ Our finding was unique suggesting that anxiety is strongly and linearly associated with insomnia, as well as its subtypes of sleep initiation difficulty and sleep maintenance difficulty. Given that the labor characteristics of truck drivers are associated with tension or worries about safety driving with time constraints, intervention for insomnia may require consideration of anxiety coping mechanism associated with driving. For this reason, future studies should be warranted in the field of labor hygiene to investigate anxiety associated with the labor characteristics of truck drivers, including their working conditions and social environments.

Our results were consistent with those of previous studies suggesting that the lifestyle profiles of truck drivers were worse than those of the general population. ${ }^{18}$ Of the 2685 truck drivers in our study, 1502 (56.7\%) were current smokers and 1027 (39.1\%) were obese (BMI $\geq 25)$. These proportions were significantly higher than those of the Japanese male general population: current smokers $(29.0 \%)$ and obese $(32.2 \%){ }^{43}$ Although past smoking was associated with insomnia, current smoking had a significant effect on sleep initiation difficulty. Tobacco is a psychostimulant that is known to disturb quality of sleep. ${ }^{44}$ Surprisingly, obesity was not associated with insomnia, including sleep maintenance difficulty which is a specific feature of OSA. ${ }^{45}$ It is known that patients with OSA mainly present with daytime sleepiness and loud snoring, rather than complaints of difficulty maintaining sleep. ${ }^{46}$ In this regard, our questionnaire did not include specific questions regarding OSA; thus, we might have missed responses of some drivers with OSA that required careful interpretation. Regarding alcohol consumption, over $253 \mathrm{mg}$ ethanol intake per week was associated with insomnia and sleep maintenance difficulty. This amount is slightly higher than the recommended $20 \mathrm{mg}$ ethanol per day by the Japanese government. ${ }^{47}$ Alcohol increases sleepiness temporarily, ${ }^{48,49}$ but it disturbs both sleep quality and quantity in the long run. ${ }^{50}$ These lifestyle factors not only disturb one's sleep but also contribute to an increased risk of lifestyle-related diseases. We also quantified the weekly caffeine intake among the subjects 
to determine its effects on insomnia. However, no significant associations were observed, unlike in previous research. $^{20}$ This may be explained by the fact that our questionnaire only asked about the type of drink instead of specific product names. Since each product contains different amounts of caffeine, these variations may have affected the non-differential misclassification. Thus, this is an apparent area for future research.

Although the linear association with driving hour category was not observed in sleep initiation difficulty, driving more than 12 hours per day had a significant effect on both insomnia and sleep maintenance difficulty. It should be noted that the number of drivers in this range of driving hours accounted for only $3.4 \%$. Given that the demand is increasing rapidly these days due to the COVID-19 pandemic, such a small percentage may not be plausible. One possible reason behind this may be the implementation of the Revised Labor Standards Act, which ordered the trucking industry to reduce drivers' workload by 2024. ${ }^{51}$ Alternatively, social desirability bias may exist. Drivers who worked long hours might have chosen responses they believe were more socially desirable or acceptable rather than choosing responses that were reflective of truth. ${ }^{52}$ For driving distance, although being a middle-haul driver had a significant effect on sleep initiation difficulty, given the insignificant effect of long-haul drive, the effect of driving area may not be robust. Considering that the driving area of each driver may suddenly change due to transportation schedules or labor shortage, the effect of driving distance may be subject to misclassification bias.

To the best of our knowledge, this survey has the largest number of truck drivers in Japan that focused on insomnia and identified anxiety, working conditions, and lifestyles as its risk factors. However, there were some limitations that should be addressed. First, the crosssectional nature of the study did not enable us to establish a cause-effect relationship. Second, information on insomnia was based on self-reported data. Thus, we could not accurately differentiate other sleep disorders such as OSA, shift work sleep disorder, and restless leg syndrome, which are also accompanied by insomnia symptoms. However, considering that drivers with insomnia symptoms are at a high risk of accidents, ${ }^{53,54}$ this limitation does not significantly affect the purpose and significance of our study. Third, given that this study was conducted in one prefecture and that nearly half of the truck drivers who were recruited did not participate in this study, generalization of the results may be limited to some extent. Moreover, because safety driving is occupational responsibility for every vehicle driver, some might hesitate to participate in the study or even after enrollment, some might be uncomfortable and thus not report their sleeping conditions. Such cases cause non-responder bias. Although our results therefore require careful interpretation, they are still valid in terms of large sample size obtained from multiple companies. Fourth, we did not include sleep duration per night in multivariable analyses. We confirmed that subjects with insomnia, and its subtypes of sleep initiation and maintenance difficulty had significantly shorter average daily sleep duration $[6.35 \pm 1.2$ hours $(p<0.001), 5.67 \pm 1.34$ hours $(p<0.001)$, and 6.46 \pm 1.15 hours $(p<0.001)$, respectively] than subjects without insomnia (6.79 \pm 1.11 hours). For truck drivers, sleep duration was thought to be relatively shorter with longer driving hours because working time plus sleeping time may be close to 24 hours. We performed a $t$-test of mean sleep duration for categories of driving hours ( $<12$ hours, $\geq 12$ hours) and found that truck drivers with longest driving hours ( $\geq 12$ hours) had significantly shorter sleep duration $(6.44 \pm 1.4$ hours) compared to other drivers $(6.75 \pm 1.1$ hours $)(p<0.04)$. Thus, it indicates that sleep duration and driving hours were highly correlated and thus we did not adjust sleep duration in the multivariable models.

\section{Conclusion}

Our observational study of 2865 Japanese truck drivers demonstrated that more than one-tenth of the subjects were suffering from insomnia and that anxiety, working conditions, and lifestyle were risk factors for insomnia. These findings suggest that further epidemiological studies are warranted to reveal the prevalence of insomnia among Japanese truck drivers based on medical diagnoses. We believe that accumulation of evidence of insomnia would lead to useful interventions, in order to stop the dangerous driving of truck drivers.

\section{Acknowledgments}

The authors would like to thank the Japan Truck Association Akita prefectural branch for technical assistance.

\section{Disclosure}

All authors declare no competing interests. 


\section{References}

1. Hege A, Perko M, Johnson A, Yu CH, Sönmez S, Apostolopoulos Y. Surveying the impact of work hours and schedules on commercial motor vehicle driver sleep. Saf Health Work. 2015;6(2):104-113. doi:10.1016/j.shaw.2015.02.001

2. Apostolopoulos Y, Sönmez S, Shattell MM, Gonzales C, Fehrenbacher C. Health survey of U.S. long-haul truck drivers: work environment, physical health, and healthcare access. Work. 2013;46(1):113-123. doi:10.3233/WOR-121553

3. Sabbagh-Ehrlich S, Friedman L, Richter ED. Working conditions and fatigue in professional truck drivers at Israeli ports. Inj Prev. 2005;11 (2):110-114. doi:10.1136/ip.2004.007682

4. Overnight bus crashes in Karuizawa, killing 14. The Japan Times. Available from: https://www.japantimes.co.jp/news/2016/01/15/ national/night-ski-bus-flips-karuizawa-three-killed-11-critical-condi tion/. Accessed January 19, 2021.

5. Ministry of Health, Labor, and Welfare. Basic survey on wage structure; 2019. Available from: https://www.e-stat.go.jp/stat-search/ files?page $=1 \&$ toukei $=00450091 \&$ tstat $=000001011429$. Accessed January 6, 2021.

6. Chen GX, Sieber WK, Lincoln JE, et al. NIOSH national survey of long-haul truck drivers: injury and safety. Accid Anal Prev. 2015;85:66-72. doi:10.1016/j.aap.2015.09.001

7. Ministry of Internal Affairs and Communications. Population census; 2015. Available from: https://www.e-stat.go.jp/stat-search/files? page $=1 \&$ toukei $=00200521$. Accessed January 6, 2021 .

8. E-commerce in the time of COVID-19 - OECD. Available from: https:// read.oecd-ilibrary.org/view/?ref=137_137212-t0fjgnerdb\&title= E-commerce-in-the-time-of-COVID-19. Accessed December 4, 2020.

9. Sagaspe P, Taillard J, Bayon V, et al. Sleepiness, near-misses and driving accidents among a representative population of French drivers. J Sleep Res. 2010;19(4):578-584. doi:10.1111/j.13652869.2009.00818.x

10. Garbarino S, Durando P, Guglielmi O, et al. Sleep apnea, sleep debt and daytime sleepiness are independently associated with road accidents. A Cross-Sectional Study on Truck Drivers. PLoS One. 2016;11 (11):e0166262. doi:10.1371/journal.pone.0166262

11. Magnavita N, Garbarino S. Sleep, health and wellness at work: a scoping review. Int $J$ Environ Res Public Health. 2017;14 (11):1347. doi:10.3390/ijerph14111347

12. National Police Agency. Traffic accident open data; 2019. Available from: https://www.npa.go.jp/publications/statistics/koutsuu/opendata/ 2019/opendata_2019.html. Accessed January 6, 2021.

13. Alhola P, Polo-Kantola P. Sleep deprivation: impact on cognitive performance. Neuropsychiatr Dis Treat. 2007;3(5):553-567.

14. Sateia MJ, Sherrill WC, Winter-Rosenberg C, Heald JL. Payer perspective of the American academy of sleep medicine clinical practice guideline for the pharmacologic treatment of chronic insomnia. $J$ Clin Sleep Med. 2017;13(2):155-157. doi:10.5664/jcsm.6428

15. Chen TY, Lee S, Buxton OM. A greater extent of insomnia symptoms and physician-recommended sleep medication use predict fall risk in community-dwelling older adults. Sleep. 2017;40(11). doi:10.1093/ sleep/zsx 142

16. Lombardi DA, Folkard S, Willetts JL, Smith GS. Daily sleep, weekly working hours, and risk of work-related injury: Us national health interview survey (2004-2008). Chronobiol Int. 2010;27 (5):1013-1030. doi:10.3109/07420528.2010.489466

17. Olfson M, Wall M, Liu SM, Morin CM, Blanco C. Insomnia and impaired quality of life in the United States. J Clin Psychiatry. 2018;79(5). doi:10.4088/JCP.17m12020

18. Guest AJ, Chen YL, Pearson N, King JA, Paine NJ, Clemes SA. Cardiometabolic risk factors and mental health status among truck drivers: a systematic review. BMJ Open. 2020;10(10):e038993. doi:10.1136/bmjopen-2020-038993
19. Magnavita N, Bortkiewicz A, Durando P, Garbarino S. Association between undiagnosed sleep disorders and cardiovascular disease in healthy truck drivers.Occup Environ Med. 2018;75(Suppl2):A63.1A63. doi:10.1136/oemed-2018-icohabstracts. 179

20. Filtness AJ, Hickman JS, Mabry JE, et al. Associations between high caffeine consumption, driving safety indicators, sleep and health behaviours in truck drivers. Saf Sci. 2020;126:104664. doi:10.1016/ j.ssci.2020.104664

21. Shattell M, Apostolopoulos Y, Snmez S, Griffin M. Occupational stressors and the mental health of truckers. Issues Ment Health Nurs. 2010;31(9):561-568. doi:10.3109/01612840.2010.488783

22. Shattell M, Apostolopoulos Y, Collins C, Sönmez S, Fehrenbacher C. Trucking organization and mental health disorders of truck drivers. Issues Ment Health Nurs. 2012;33(7):436-444. doi:10.3109/ 01612840.2012 .665156

23. Orris P, Hartman DE, Strauss $\mathrm{P}$, et al. Stress among package truck drivers. Am J Ind Med. 1997;31(2):202-210. doi:10.1002/(SICI) 1097-0274(199702)31:2<202::AID-AJIM10>3.0.CO;2-5

24. Qiu L, Nixon WA. Effects of adverse weather on traffic crashes. Transp Res Rec J Transp Res Board. 2008;2055(1):139-146. doi:10.3141/2055-16

25. Wong WCW, Tam SM, Leung PWS. Cross-border truck drivers in hong kong: their psychological health, sexual dysfunctions and sexual risk behaviors. J Travel Med. 2007;14(1):20-30. doi:10.1111/ j.1708-8305.2006.00085.x

26. Garbarino S, Guglielmi O, Sannita WG, Magnavita N, Lanteri P. Sleep and mental health in truck drivers: descriptive review of the current evidence and proposal of strategies for primary prevention. Int J Environ Res Public Health. 2018;15(9):1852. doi:10.3390/ ijerph15091852

27. National Census Japan; 2015. Available from: https://www.e-stat.go. jp/dbview?sid=0003209820. Accessed January 31, 2021.

28. International classification of sleep disorders - third edition (ICSD-3) (Online). Available from: https://learn.aasm.org/Public/Catalog/ Details.aspx?id=\%2FgqQVDMQIT\%2FEDy $86 \mathrm{PWgqgQ} \% 3 \mathrm{D} \%$ $3 \mathrm{D} \&$ returnurl $=\% 2 \mathrm{FU}$ sers $\% 2 \mathrm{FUserOnlineCourse.aspx} \%$ 3 F Learning Activity I D \% 3 D \% 252 fgq Q V D M Q I T \% 252fEDy86PWgqgQ\%253d\%253d. Accessed November 26, 2020.

29. Yokoyama E, Kaneita Y, Saito Y, et al. Association between depression and insomnia subtypes: a longitudinal study on the elderly in Japan. Sleep. 2010;33(12):1693-1702. doi:10.1093/sleep/33.12.1693

30. Bjorøy I, Jørgensen VA, Pallesen S, Bjorvatn B. The prevalence of insomnia subtypes in relation to demographic characteristics, anxiety, depression, alcohol consumption and use of hypnotics. Front Psychol. 2020;11. doi:10.3389/fpsyg.2020.00527

31. Spielberger CD, Gorsuch RL, Lushene RE STAI manual for the State-trait anxiety inventory ("self-evaluation questionnaire"); 1970. Available from: https://ci.nii.ac.jp/ncid/BA66835301. Accessed November 26, 2020.

32. Nakazato K, Mizuguchi T. Development and validation of Japanese version of state-trait anxiety inventory-A study with female subjects. Jpn J Psychosom Med. 1982;22:107-112.

33. Fact sheets. Food Safety Commission of Japan; 2018. Available from: https://www.fsc.go.jp/factsheets/index.data/factsheets_caffeine.pdf. Accessed January 30, 2021.

34. Nawrot P, Jordan S, Eastwood J, Rotstein J, Hugenholtz A, Feeley M. Effects of caffeine on human health. Food Addit Contam. 2003;20 (1):1-30. doi:10.1080/0265203021000007840

35. Itani O, Kaneita Y, Munezawa T, et al. Nationwide epidemiological study of insomnia in Japan. Sleep Med. 2016;25:130-138. doi:10.1016/j.sleep.2016.05.013

36. Sunwoo JS, Shin DS, Hwangbo Y, et al. High risk of obstructive sleep apnea, insomnia, and daytime sleepiness among commercial motor vehicle drivers. Sleep Breath. 2019;23(3):979-985. doi:10.1007/s11325-019-01805-7 
37. Manual on obstructive sleep apnea prevention for road freight companies. Ministry of Land, Infrastructure and Transport; 2015.

38. Ueyama M, Kokuto H, Sugihara H, et al. Investigation of obstructive sleep apnea using portable monitors and health check data in Japanese drivers. J Atheroscler Thromb. 2018;25(11):1118-1127. doi: $10.5551 /$ jat. 41806

39. Cui R, Tanigawa T, Sakurai S, Yamagishi K, Iso H. Relationships between sleep-disordered breathing and blood pressure and excessive daytime sleepiness among truck drivers. Hypertens Res. 2006;29 (8):605-610. doi:10.1291/hypres.29.605

40. Garbarino S, Magnavita N. Sleep problems are a strong predictor of stress-related metabolic changes in police officers. A prospective study. PLoS One. 2019;14(10):e224259. doi:10.1371/journal. pone. 0224259

41. Grupe DW, Nitschke JB. Uncertainty and anticipation in anxiety: an integrated neurobiological and psychological perspective. Nat Rev Neurosci. 2013;14(7):488-501. doi:10.1038/nrn3524

42. Iwata N, Mishima N, Shimizu T, et al. The Japanese adaptation of the STAI form Y in Japanese working adults - the presence or absence of anxiety. Ind Health. 1998;36(1):8-13. doi:10.2486/indhealth.36.8

43. National health and nutrition survey 2019. Ministry of Health, Labor and Welfare. Available from: https://www.mhlw.go.jp/stf/newpage 08789.html. Accessed January 25, 2021.

44. Zhang L, Samet J, Caffo B, Punjabi NM. Cigarette smoking and nocturnal sleep architecture. Am J Epidemiol. 2006;164(6):529-537. doi:10.1093/aje/kwj231

45. Leinum CJ, Dopp JM, Morgan BJ. Sleep-disordered breathing and obesity: pathophysiology, complications, and treatment. Nutr Clin Pract. 2009;24(6):675-687. doi:10.1177/0884533609351532

46. Cho YW, Kim KT, Moon HJ, Korostyshevskiy VR, Motamedi GK, Yang KI. Comorbid insomnia with obstructive sleep apnea: clinical characteristics and risk factors. J Clin Sleep Med. 2018;14 (3):409-417. doi:10.5664/jcsm.6988
47. Recommended alcohol consumption. Ministry of Health, Labor and Welfare. Available from: https://www.mhlw.go.jp/www1/topics/ kenko21_11/b5.html. Accessed January 25, 2021.

48. Arnedt JT, Wilde GJS, Munt PW, MacLean AW. How do prolonged wakefulness and alcohol compare in the decrements they produce on a simulated driving task? Accid Anal Prev. 2001;33(3):337-344. doi:10.1016/S0001-4575(00)00047-6

49. Rohsenow DJ, Howland J, Minsky SJ, Arnedt JT. Effects of heavy drinking by maritime academy cadets on hangover, perceived sleep, and next-day ship power plant operation. $J$ Stud Alcohol. 2006;67 (3):406-415. doi:10.15288/jsa.2006.67.406

50. Colrain IM, Nicholas CL, Baker FC. Alcohol and the sleeping brain. In: Handbook of Clinical Neurology. Vol. 125. Elsevier B.V.; 2014:415-431. doi:10.1016/B978-0-444-62619-6.00024-0

51. Revised Labour Standards Act. Ministry of Health, Labor and Welfare. Available from: https://www.mhlw.go.jp/stf/seisakunitsuite/ bunya/0000148322 00037.html. Accessed January 25, 2021.

52. Latkin CA, Edwards C, Davey-Rothwell MA, Tobin KE. The relationship between social desirability bias and self-reports of health, substance use, and social network factors among urban substance users in Baltimore, Maryland. Addict Behav. 2017;73:133-136. doi:10.1016/j.addbeh.2017.05.005

53. Laugsand LE, Strand LB, Vatten LJ, Janszky I, Bjørngaard JH. Insomnia symptoms and risk for unintentional fatal injuries- the HUNT Study. Sleep. 2014;37(11):1777-1786. doi:10.5665/ sleep. 4170

54. Garbarino S, Magnavita N, Guglielmi O, et al. Insomnia is associated with road accidents. Further evidence from a study on truck drivers. PLoS One. 2017;12(10):e0187256. doi:10.1371/journal. pone. 0187256

\section{Publish your work in this journal}

Nature and Science of Sleep is an international, peer-reviewed, open access journal covering all aspects of sleep science and sleep medicine, including the neurophysiology and functions of sleep, the genetics of sleep, sleep and society, biological rhythms, dreaming, sleep disorders and therapy, and strategies to optimize healthy sleep.
The manuscript management system is completely online and includes a very quick and fair peer-review system, which is all easy to use. Visit http://www.dovepress.com/testimonials.php to read real quotes from published authors. 